\title{
A normal criterion concerning zero numbers
}

\author{
Chengxiong Sun ${ }^{1}$
}

Received: 4 March 2021 / Accepted: 4 June 2021 / Published online: 17 November 2021

(c) The Author(s), under exclusive licence to Springer-Verlag Italia S.r.l., part of Springer Nature 2021

\begin{abstract}
Let $n \geq 4$ be a positive integer, $\mathcal{F}$ be a family of meromorphic functions in $D$ and let $a(z)(\not \equiv 0), b(z)$ be two holomorphic functions in $D$. If, for any function $f \in \mathcal{F},(1) f(z) \neq \infty$ when $a(z)=0$, (2) $f^{\prime}(z)-a(z) f^{n}(z)-b(z)$ has at most one zero in $D$, then $\mathcal{F}$ is normal in D.
\end{abstract}

Keywords Meromorphic function · Normal family $\cdot$ Shared value

Mathematics Subject Classification Primary 30D45

\section{Introduction and main results}

Let $D$ be a domain in $\mathbb{C}$ and $\mathcal{F}$ be a family of meromorphic functions in $D$. A family $\mathcal{F}$ is said to be normal in $D$, in the sense of Montel, if each sequence $\left\{f_{n}\right\}$ has a subsequence $\left\{f_{n_{k}}\right\}$ that converges spherically locally uniformly in $D$ to a meromorphic function or to the constant $\infty$.

The following well-known normal conjecture was proposed by Hayman in 1967.

Theorem A [1] Let $n \geq 5$ be a positive integer, $\mathcal{F}$ be a family of meromorphic functions in $D$ and let $a(\neq 0), b$ be two finite complex numbers. If, for any function $f \in \mathcal{F}, f^{\prime}(z)-a f^{n}(z) \neq b$ in $D$, then $\mathcal{F}$ is normal in $D$.

The conjecture was proved by $\mathrm{Li}$ [2], Li [3], Langley [4] (for $n \geq 5$ ), Pang [5] (for $n=4)$, Chen and Fang [6], Bergweiler and Eremenko [7] (for $n=3$ ).

Up to now, this result has undergone various extensions and improvements (see [8-12]).

The following generalization of Theorem A was proved by Yang et al. [13].

Theorem B Let $n \geq 4$ be a positive integer, $\mathcal{F}$ be a family of meromorphic functions in $D$ and let $a(z)(\equiv 0), b(z)$ be two holomorphic functions in $D$. If, for any function $f \in \mathcal{F}$, (1) $f(z) \neq \infty$ when $a(z)=0,(2) f^{\prime}(z)-a(z) f^{n}(z) \neq b(z)$ in $D$, then $\mathcal{F}$ is normal in $D$.

Chengxiong Sun

aatram@aliyun.com

1 Institute of Mathematics, School of Mathematics, Yunnan, People's Republic of China 
It is natural to ask what can be said if $f^{\prime}(z)-a(z) f^{n}(z)=b(z)$ has solutions in Theorem B.

In this article, we study this problem and prove the following result.

Theorem 1.1 Let $n \geq 4$ be a positive integer, $\mathcal{F}$ be a family of meromorphic functions in $D$ and let $a(z)(\equiv \equiv), b(z)$ be two holomorphic functions in $D$. If, for any function $f \in \mathcal{F}$, (1) $f(z) \neq \infty$ when $a(z)=0,(2) f^{\prime}(z)-a(z) f^{n}(z)-b(z)$ has at most one zero in $D$, then $\mathcal{F}$ is normal in $D$.

The following examples show that both $n \geq 4$ and the conditions (1), (2) in Theorem 1.1 are necessary and sharp.

Example 1.1 Let $\Delta=\{z:|z|<1\}$ and $a(z)=-2 z^{3}, b(z)=0$. Let $\mathcal{F}=\left\{f_{j}(z)\right\}$, where

$$
f_{j}(z)=\frac{1}{z^{2}-\frac{1}{j}}, z \in \Delta, j=1,2 \ldots .
$$

Then $f_{j}^{\prime}(z)-a(z) f_{j}^{3}(z)-b(z)=\frac{\frac{2}{j} z}{\left(z^{2}-\frac{1}{j}\right)^{3}}$, which has a zero in $\Delta$. Clearly, $f \neq \infty$ when $a(z)=0$, however $\mathcal{F}$ is not normal at 0 .

Example 1.2 Let $\Delta=\{z:|z|<1\}, a(z)=-z, b(z)=0$. Let $\mathcal{F}=\left\{f_{j}(z)\right\}$, where

$$
f_{j}(z)=\frac{1}{j+z}, z \in \Delta, j=1,2 \ldots
$$

Then $f_{j}^{\prime}(z)-a(z) f_{j}^{4}(z)-b(z)=\frac{-z^{2}+(1-2 j) z-j^{2}}{(j+z)^{4}}$, which has exactly two distinct zeros in $\Delta$. Clearly, $f \neq \infty$ when $a(z)=0$, however $\mathcal{F}$ is not normal at 0 .

Example 1.3 Let $\Delta=\{z:|z|<1\}, a(z)=-z, b(z)=0$. Let $\mathcal{F}=\left\{f_{j}(z)\right\}$, where

$$
f_{j}(z)=\frac{1}{j z}, z \in \Delta, j=2,3 \ldots
$$

Then $f_{j}^{\prime}(z)-a(z) f_{j}^{4}(z)-b(z)=\frac{1-j^{3} z}{j^{4} z^{3}}$, which has a zero in $\Delta$. But $\mathcal{F}$ is not normal at 0 .

The next example shows that Theorem 1.1 is not valid if $a(z)$ is a meromorphic function in $D$.

Example 1.4 Let $\Delta=\{z:|z|<1\}, a(z)=z^{-4}, b(z)=0$. Let $\mathcal{F}=\left\{f_{j}(z)\right\}$, where

$$
f_{j}(z)=j z, z \in \Delta, j=2,3 \ldots
$$

Then $f_{j}^{\prime}(z)-a(z) f_{j}^{4}(z)-b(z)=j-j^{4}$, which has no zero in $\Delta$. But $\mathcal{F}$ is not normal at 0 .

Remark 1.1 Clearly Theorem 1.1 improves and extends Theorem B. 


\section{Some lemmas}

Lemma 2.1 [14] Let $k$ be a positive integer and let $\mathcal{F}$ be a family of functions meromorphic in the unit disc $\Delta$, all of whose zeros have multiplicity at least $k$. If $\mathcal{F}$ is not normal in any neighbourhood of $z_{0} \in \Delta$, then for each $\alpha, 0 \leq \alpha<k$ there exist a sequence of complex numbers $z_{n}, z_{n} \rightarrow z_{0}, z_{0} \in \Delta$, a sequence of positive numbers $\rho_{n} \rightarrow 0$, and a sequence of functions $f_{n} \in \mathcal{F}$ such that $g_{n}(\xi)=\rho_{n}^{-\alpha} f_{n}\left(z_{n}+\rho_{n} \xi\right) \rightarrow g(\xi)$ spherically uniformly on compact subsets of $\mathbb{C}$, where $g$ is a non-constant meromorphic function, all of whose zeros have multiplicity at least $k$. Moreover, $g(\xi)$ has order at most 2 .

We remark that one can take $-k<\alpha \leq 0$ in the above lemma if all poles of each $f_{n} \in \mathcal{F}$ have multiplicity at least $k$ (see [15]).

Lemma 2.2 [16, Theorem 4, p. 381] Let $f(z)$ be a transcendental meromorphic function with finite order, all of whose zeros are of multiplicity at least 2 , and let $P(z)(\equiv \equiv 0)$ be a polynomial, then $f^{\prime}(z)-P(z)$ has infinitely many zeros.

Lemma 2.3 [17, Lemma 6] Let $k$ be a positive integer, $f(z)$ be a transcendental meromorphic function with finite order, all of whose zeros have multiplicity at least $k+1$, then $f^{(k)}-1$ has infinitely many zeros.

Lemma 2.4 [18, Lemma 5] Let $k$ be a positive integer, $f(z)$ be a nonconstant rational function, all of whose zeros have multiplicity at least $k+2$, and all of whose poles have multiplicity at least 3 , then $f^{(k)}(z)-1$ has at least two distinct zeros.

Lemma 2.5 [12, Lemma 3] Let $n \geq 4$ be a positive integer, $a \neq 0$ be a finite complex number and let $f(z)$ be a nonconstant meromorphic function, then $f^{\prime}(z)-a f^{n}(z)$ has at least two distinct zeros.

Lemma 2.6 [19, Lemma 7(iv), p. 261] Let $k$, $l$ be two positive integers, $Q(z)$ be a rational function, all of whose zeros have multiplicity at least $k+2$ and all of whose poles have multiplicity at least 2 with the possible exception of $z=0$, then $Q^{(k)}(z)=z^{l}$ has a solution in $\mathbb{C}$.

Lemma 2.7 [20, Lemma 11] Let $k, m$ be two positive integers, $f(z)$ be a rational function. If $f(z) \neq 0$ for $z \in \mathbb{C}$, and $f^{(k)}(z) \neq z^{m}$ for $z \neq z_{0}$, where $z_{0} \in \mathbb{C}$, then $f(z)$ is a constant.

Lemma 2.8 Let $n \geq 4$ be a positive integer, $\mathcal{F}=\left\{f_{j}\right\}$ be a family of meromorphic functions in a domain $D$, and let $a_{j}(z), b_{j}(z)$ be two sequences of analytic functions in $D$ such that $a_{j}(z) \longrightarrow a(z) \neq 0, b_{j}(z) \longrightarrow b(z)$. If $f_{j}^{\prime}(z)-a_{j}(z) f_{j}^{n}(z)-b_{j}(z)$ has at most one zero, then $\mathcal{F}$ is normal in $D$.

Proof Suppose that $\mathcal{F}$ is not normal at $z_{0} \in D$. By Lemma 2.1, there exists $z_{j} \rightarrow z_{0}, \rho_{j} \rightarrow 0^{+}$, and $f_{j} \in \mathcal{F}$ such that

$$
g_{j}(\xi)=\rho_{j}^{\frac{1}{n-1}} f_{j}\left(z_{j}+\rho_{j} \xi\right) \longrightarrow g(\xi)
$$


locally uniformly on compact subsets of $\mathbb{C}$, where $g(\xi)$ is a non-constant meromorphic function in $\mathbb{C}$.

For each ${ }_{n} \quad \xi \in \mathbb{C} /\left\{g^{-1}(\infty)\right\}, \quad$ we have $g_{j}^{\prime}(\xi)-a_{j}\left(z_{j}+\rho_{j} \xi\right) g_{j}^{n}(\xi)-\rho_{j}^{\frac{n}{n-1}} b_{j}\left(z_{j}+\rho_{j} \xi\right) \longrightarrow g^{\prime}(\xi)-a\left(z_{0}\right) g^{n}(\xi)$.

Obviously, $g^{\prime}(\xi)-a\left(z_{0}\right) g^{n}(\xi) \not \equiv 0$.

Suppose that $g^{\prime}(\xi)-a\left(z_{0}\right) g^{n}(\xi) \equiv 0$, then $g(\xi)$ must be an entire function. Hence, since $n \geq 3$ and $a\left(z_{0}\right) \neq 0$, we have

$$
n T(r, g)=T\left(r, g^{n}\right)=T\left(r, \frac{g^{\prime}}{a\left(z_{0}\right)}\right)=T\left(r, g^{\prime}\right)+\log ^{+} \frac{1}{\left|a\left(z_{0}\right)\right|} \leq T(r, g)+S(r, g) .
$$

It follows that we have $T(r, g)=S(r, g)$. But this is impossible since $g(\xi)$ is a non-constant meromorphic function.

Claim: $g^{\prime}(\xi)-a\left(z_{0}\right) g^{n}(\xi)$ has at most one zero.

Suppose this is not the case, and $g^{\prime}(\xi)-a\left(z_{0}\right) g^{n}(\xi)$ has two distinct zeros $\xi_{1}$, and $\xi_{2}$. We choose a positive number $\delta$ small enough such that $D_{1} \cap D_{2}=\emptyset$ and $g^{\prime}(\xi)-a\left(z_{0}\right) g^{n}(\xi)$ has no other zeros in $D_{1} \cup D_{2}$ except for $\xi_{1}$ and $\xi_{2}$, where $D_{1}=\left\{\xi:\left|\xi-\xi_{1}\right|<\delta\right\}$ and $D_{2}=\left\{\xi:\left|\xi-\xi_{2}\right|<\delta\right\}$.

By Hurwitz's theorem, for sufficiently large $j$, there exist points $\xi_{1, j} \rightarrow \xi_{1}$ and $\xi_{2, j} \rightarrow \xi_{2}$ such that

$$
f_{j}^{\prime}\left(\xi_{1, j}\right)-a_{j}\left(z_{j}+\rho_{j} \xi_{1, j}\right) f_{j}^{n}\left(z_{j}+\rho_{j} \xi_{1, j}\right)-b_{j}\left(z_{j}+\rho_{j} \xi_{1, j}\right)=0
$$

and

$$
f_{j}^{\prime}\left(\xi_{2, j}\right)-a_{j}\left(z_{j}+\rho_{j} \xi_{2, j}\right) f_{j}^{n}\left(z_{j}+\rho_{j} \xi_{2, j}\right)-b_{j}\left(z_{j}+\rho_{j} \xi_{2, j}\right)=0
$$

Since $f_{j}^{\prime}(z)-a_{j}(z) f_{j}^{n}(z)-b_{j}(z)$ has at most one zero in $D$, then

$$
z_{j}+\rho_{j} \xi_{1, j}=z_{j}+\rho_{j} \xi_{2, j}
$$

this is

$$
\xi_{1, j}=\xi_{2, j}=\frac{z_{0}-z_{j}}{\rho_{j}},
$$

which contradicts the fact $D_{1} \cap D_{2}=\emptyset$. The claim is proved.

From Lemma 2.5, we have $g^{\prime}(\xi)-a\left(z_{0}\right) g^{n}(\xi)$ has at least two distinct zeros, this contradicts Claim which says that $g^{\prime}(\xi)-a\left(z_{0}\right) g^{n}(\xi)$ has at most one zero. Therefore $\mathcal{F}$ is normal in $D$.

\section{Proof of theorems}

Proof of Theorem 1.1 Suppose that $\mathcal{F}$ is not normal at $z_{0}$. From Lemma 2.8, we obtain $a\left(z_{0}\right)=0$. Without loss of generality, we assume that $z_{0}=0$ and $a(z)=z^{m} \phi(z)$, where $m \in \mathbb{N}$, and $\phi$ is holomorphic with $\phi(0) \neq 0$. We can say $\phi \neq 0$ in $\Delta\left(0, \delta_{0}\right)=\left\{z:|z|<\delta_{0}\right\}$ with the normalization $\phi(0)=1$.

Since $f(z) \neq \infty$ when $a(z)=0$ and $f^{\prime}(z)-a(z) f^{n}(z)-b(z)$ has at most one zero in $\Delta$, then $\frac{f^{\prime}(z)}{a(z) f^{n}(z)}-\frac{b(z)}{a(z) f^{n}(z)}-1$ has at most one zero in $\Delta$. Consider the family as follows 


$$
\mathcal{G}=\left\{g(z)=\frac{1}{a(z) f^{n-1}(z)}, f \in \mathcal{F}\right\},
$$

where all zeros and poles of $g(z)$ have multiplicity at least $n-1(\geq 3)$, except possibly the pole at 0 , which has order at least $m$. Hence $\mathcal{G}$ is not normal at $z_{0}=0$ in $\Delta$. Then, by Lemma 2.1, there exists $z_{j} \longrightarrow 0, g_{j} \in \mathcal{G}$ and $\rho_{j} \longrightarrow 0^{+}$such that

$$
G_{j}(\xi)=\frac{g_{j}\left(z_{j}+\rho_{j} \xi\right)}{\rho_{j}} \longrightarrow G(\xi)
$$

locally uniformly on compact subsets of $\mathbb{C}$, where $G(\xi)$ is a non-constant meromorphic functions in $\mathbb{C}$, and all zeros and poles of $G(\xi)$ have multiplicity at least $n-1(\geq 3)$, except possibly a pole, which has order at least $m$.

Next we consider two cases according to whether the sequence $\frac{z_{n}}{\rho_{n}}$ is bounded or unbounded.

Case 1. First assume that the sequence $\frac{z_{n}}{\rho_{n}}$ is unbounded. Then, there exists a subsequence, which we continue to call $\frac{z_{n}}{\rho_{n}}$, such that $\frac{\underline{\rho}_{n}}{\rho_{n}} \rightarrow \infty$.

By simple calculation, from (3.1) and (3.2), we have

$$
\begin{aligned}
G_{j}^{\prime}(\xi)= & {\left[\frac{g_{j}\left(z_{j}+\rho_{j} \xi\right)}{\rho_{j}}\right]^{\prime}=\left[\frac{1}{\rho_{j} a\left(z_{j}+\rho_{j} \xi\right) f_{j}^{n-1}\left(z_{j}+\rho_{j} \xi\right)}\right]^{\prime} } \\
= & -\frac{a^{\prime}\left(z_{j}+\rho_{j} \xi\right)}{a^{2}\left(z_{j}+\rho_{j} \xi\right) f_{j}^{n-1}\left(z_{j}+\rho_{j} \xi\right)}-\frac{(n-1) f_{j}^{\prime}\left(z_{j}+\rho_{j} \xi\right)}{a\left(z_{j}+\rho_{j} \xi\right) f_{j}^{n}\left(z_{j}+\rho_{j} \xi\right)} \\
= & -\frac{a^{\prime}\left(z_{j}+\rho_{j} \xi\right)}{a^{2}\left(z_{j}+\rho_{j} \xi\right) f_{j}^{n-1}\left(z_{j}+\rho_{j} \xi\right)}-\frac{(n-1) b\left(z_{j}+\rho_{j} \xi\right)}{a\left(z_{j}+\rho_{j} \xi\right) f_{j}^{n}\left(z_{j}+\rho_{j} \xi\right)} \\
& -(n-1)\left[\frac{f_{j}^{\prime}\left(z_{j}+\rho_{j} \xi\right)}{a\left(z_{j}+\rho_{j} \xi\right) f_{j}^{n}\left(z_{j}+\rho_{j} \xi\right)}-\frac{b\left(z_{j}+\rho_{j} \xi\right)}{a\left(z_{j}+\rho_{j} \xi\right) f_{j}^{n}\left(z_{j}+\rho_{j} \xi\right)}\right] .
\end{aligned}
$$

Noting that 


$$
\begin{aligned}
& \frac{a^{\prime}\left(z_{j}+\rho_{j} \xi\right)}{a^{2}\left(z_{j}+\rho_{j} \xi\right) f_{j}^{n-1}\left(z_{j}+\rho_{j} \xi\right)} \\
& =\frac{m\left(z_{j}+\rho_{j} \xi\right)^{m-1} \rho_{j} \phi\left(z_{j}+\rho_{j} \xi\right)+\left(z_{j}+\rho_{j} \xi\right)^{m} \rho_{j} \phi^{\prime}\left(z_{j}+\rho_{j} \xi\right)}{\left(z_{j}+\rho_{j} \xi\right)^{2 m} \phi^{2}\left(z_{j}+\rho_{j} \xi\right)} \\
& \quad g_{j}\left(z_{j}+\rho_{j} \xi\right)\left(z_{j}+\rho_{j} \xi\right)^{m} \phi\left(z_{j}+\rho_{j} \xi\right) \\
& =\frac{m \rho_{j}}{z_{j}+\rho_{j} \xi} g_{j}\left(z_{j}+\rho_{j} \xi\right)+\frac{\rho_{j} \phi^{\prime}\left(z_{j}+\rho_{j} \xi\right)}{\phi\left(z_{j}+\rho_{j} \xi\right)} g_{j}\left(z_{j}+\rho_{j} \xi\right) \rightarrow 0, \text { and } \\
& \left.\frac{b\left(z_{j}+\rho_{j} \xi\right)}{a\left(z_{j}+\rho_{j} \xi\right) f_{j}^{n}\left(z_{j}+\rho_{j} \xi\right)}\right]^{n-1} \\
& =\rho_{j}^{n} a\left(z_{j}+\rho_{j} \xi\right) b^{n-1}\left(z_{j}+\rho_{j} \xi\right)\left[\frac{1}{\rho_{j} a\left(z_{j}+\rho_{j} \xi\right) f_{j}^{n-1}\left(z_{j}+\rho_{j} \xi\right)}\right]^{n} \\
& =\rho_{j}^{n} a\left(z_{j}+\rho_{j} \xi\right) b^{n-1}\left(z_{j}+\rho_{j} \xi\right) G_{j}^{n}(\xi) \rightarrow 0
\end{aligned}
$$

uniformly on compact subsets of $\mathbb{C}$ disjoint from the poles of $G$. we deduce that $-(n-1)\left[\frac{f_{j}^{\prime}\left(z_{j}+\rho_{j} \xi\right)}{a\left(z_{j}+\rho_{j} \xi\right) f_{j}^{n}\left(z_{j}+\rho_{j} \xi\right)}-\frac{b\left(z_{j}+\rho_{j} \xi\right)}{a\left(z_{j}+\rho_{j} \xi\right) f_{j}^{n}\left(z_{j}+\rho_{j} \xi\right)}\right] \rightarrow G^{\prime}(\xi)$

uniformly on compact subsets of $\mathbb{C}$ disjoint from the poles of $G$.

If $G^{\prime}(\xi) \equiv-(n-1)=1-n$, then $G(\xi)=(1-n) \xi+A$, where $A$ is a constant, which contradicts the fact that all zeros of $G(\xi)$ have multiplicity at least $n-1$. Hence, we have $G^{\prime}(\xi) \not \equiv-(n-1)$.

Using an argument similar to Claim in Lemma 2.8, we can obtain $G^{\prime}(\xi)+(n-1)$ has at most one zero.

On the other hand, by Lemmas 2.3 and 2.4, we have $G^{\prime}(\xi)+(n-1)$ has at least two distinct zeros. Hence $G(\xi)$ is a constant, a contradiction.

Case 2. Now we consider the case that $\frac{z_{n}}{\rho_{n}}$ is bounded. Then, there is a subsequence, which we continue to call $\frac{z_{n}}{\rho_{n}}$, such that $\frac{z_{n}}{\rho_{n}} \rightarrow \alpha \in \mathbb{C}$.

It follows from (3.2) that

$$
\frac{g_{j}\left(\rho_{j} \xi\right)}{\rho_{j}}=\frac{g_{j}\left(z_{j}+\rho_{j}\left(\xi-\frac{z_{j}}{\rho_{j}}\right)\right)}{\rho_{j}}=G_{j}\left(\xi-\frac{z_{j}}{\rho_{j}}\right) \rightarrow G(\xi-\alpha)=\widehat{G}(\xi)
$$

spherically uniformly on compact subsets of $\mathbb{C}$. Clearly, all zeros and all poles of $\widehat{G}(\xi)$ have multiplicity at least $n-1$, and $\xi=0$ is a pole of $\widehat{G}(\xi)$ with multiplicity at least $m$.

Set

$$
H_{j}(\xi)=\frac{1}{\rho_{j}^{m+1} f_{j}^{n-1}\left(\rho_{j} \xi\right)} .
$$

It follows from (3.2) and (3.3) that

$$
H_{j}(\xi)=a\left(\rho_{j} \xi\right) \frac{g_{j}\left(z_{j}+\rho_{j}\left(\xi-\frac{z_{j}}{\rho_{j}}\right)\right)}{\rho_{j}} \rightarrow H(\xi)=\xi^{m} G(\xi-\alpha)=\xi^{m} \widehat{G}(\xi)
$$


spherically locally uniformly on $\mathbb{C} \backslash\{0\}$, or on $\mathbb{C}$ if $G(-\alpha) \neq \infty$. Obviously, all zeros and all poles of $H(\xi)$ have multiplicity at least $n-1$, and $H(0) \neq 0$ since $f(\xi) \neq \infty$ when $a(\xi)=0$.

Noting that

$$
\begin{aligned}
& H_{j}^{\prime}(\xi)+(n-1) \frac{b\left(\rho_{j} \xi\right)}{\rho_{j}^{m} f_{j}^{n}\left(\rho_{j} \xi\right)}=\left[\frac{1}{\rho_{j}^{m+1} f_{j}^{n-1}\left(\rho_{j} \xi\right)}\right]^{\prime}+(n-1) \frac{b\left(\rho_{j} \xi\right)}{\rho_{j}^{m} f_{j}^{n}\left(\rho_{j} \xi\right)} \\
& \quad=-(n-1) \xi^{m} \phi\left(\rho_{j} \xi\right)\left[\frac{f_{j}^{\prime}\left(\rho_{j} \xi\right)}{a\left(\rho_{j} \xi\right) f_{j}^{n}\left(\rho_{j} \xi\right)}-\frac{b\left(\rho_{j} \xi\right)}{a\left(\rho_{j} \xi\right) f_{j}^{n}\left(\rho_{j} \xi\right)}\right] .
\end{aligned}
$$

On the other hand,

$$
\begin{aligned}
& {\left[\frac{b\left(\rho_{j} \xi\right)}{\rho_{j}^{m} f_{j}^{n}\left(\rho_{j} \xi\right)}\right]^{n-1}=\frac{\rho_{j}^{n} b^{n-1}\left(\rho_{j} \xi\right) a^{n}\left(\rho_{j} \xi\right)}{\rho_{j}^{m(n-1)}} \frac{1}{\rho_{j}^{n} a^{n}\left(\rho_{j} \xi\right) f_{j}^{n(n-1)}\left(\rho_{j} \xi\right)}} \\
& \quad=\frac{\rho_{j}^{n} b^{n-1}\left(\rho_{j} \xi\right) a^{n}\left(\rho_{j} \xi\right)}{\rho_{j}^{m(n-1)}} G_{j}^{n}\left(\rho_{j} \xi\right)=\frac{\rho_{j}^{n} b^{n-1}\left(\rho_{j} \xi\right) a^{n}\left(\rho_{j} \xi\right)}{\rho_{j}^{m(n-1)}} G_{j}^{n}\left(z_{j}+\rho_{j}\left(\xi-\frac{z_{j}}{\rho_{j}}\right)\right) \\
& \quad=\frac{\rho_{j}^{n(m+1)} b^{n-1}\left(\rho_{j} \xi\right) \xi^{n m} \phi^{n}\left(\rho_{j} \xi\right)}{\rho_{j}^{m(n-1)}} G_{j}^{n}\left(z_{j}+\rho_{j}\left(\xi-\frac{z_{j}}{\rho_{j}}\right)\right) \\
& =\rho_{j}^{m+n} b^{n-1}\left(\rho_{j} \xi\right) \xi^{n m} \phi^{n}\left(\rho_{j} \xi\right) G_{j}^{n}\left(z_{j}+\rho_{j}\left(\xi-\frac{z_{j}}{\rho_{j}}\right)\right) \rightarrow 0
\end{aligned}
$$

uniformly on compact subsets of $\mathbb{C}$ disjoint from the poles of $G$. Hence

$$
-(n-1) \xi^{m} \phi\left(\rho_{j} \xi\right)\left[\frac{f_{j}^{\prime}\left(\rho_{j} \xi\right)}{a\left(\rho_{j} \xi\right) f_{j}^{n}\left(\rho_{j} \xi\right)}-\frac{b\left(\rho_{j} \xi\right)}{a\left(\rho_{j} \xi\right) f_{j}^{n}\left(\rho_{j} \xi\right)}\right] \rightarrow H^{\prime}(\xi)
$$

uniformly on compact subsets of $\mathbb{C}$ disjoint from the poles of $G$.

If $H^{\prime}(\xi) \equiv-(n-1) \xi^{m}$, then $H(\xi)=\frac{(1-n) \xi^{m+1}}{m+1}+B$, where $B$ is a constant. Thus, $H(\xi)$ has at least one zero in $\mathbb{C}$. Let $\xi_{0}$ be a zero of $H(\xi)$, then by the fact that $\xi_{0}$ has multiplicity at least $n-1(\geq 3)$, we get $H^{\prime}\left(\xi_{0}\right)=0$ and hence $\xi_{0}=0$ by $H^{\prime}(\xi) \equiv-(n-1) \xi^{m}$. It follows that $H(\xi)=c \xi^{l}, c \in \mathbb{C}$ and $l \in \mathbb{N}$. Thus, $\frac{(1-n) \xi^{m+1}}{m+1}+B=c \xi^{l}$. By comparing the degrees and coefficients, we see that $B=0$. Thus, $H(\xi) \stackrel{m+1}{=} \frac{(1-n) \xi^{m+1}}{m+1}$. It follows that $H(0)=0$, which contradicts that $H(0) \neq 0$.

Using an argument similar to Claim in Lemma 2.8, we can obtain $H^{\prime}(\xi)+(n-1) \xi^{m}$ has at most one zero.

Next we consider two subcases according to whether $H^{\prime}(\xi)+(n-1) \xi^{m}$ has zero or not.

Case 2.1 $H^{\prime}(\xi) \neq(n-1) \xi^{m}$. It now follows from Lemmas 2.2 and 2.6 that Case 2.1 cannot occur.

Case 2.2 $H^{\prime}(\xi)=(n-1) \xi^{m}$. Then $H^{\prime}(\xi) \neq(n-1) \xi^{m}$ for $\xi \neq \xi_{0}$, where $\xi_{0} \in \mathbb{C}$.

Case 2.2.1 $\xi_{0}=0$. Thus $H^{\prime}(0)=0$. It follows that $H(0)=0$, which contradicts that $H(0) \neq 0$.

Case 2.2.2 $\xi_{0} \neq 0$.

If $H\left(\xi_{0}\right)=0$, then $H^{\prime}\left(\xi_{0}\right)=0$ since all zeros of $H(\xi)$ have multiplicity at least $n-1$. Thus $\xi_{0}=0$, which contradicts that $\xi_{0} \neq 0$. 
If $H(\xi) \neq 0, \xi \in \mathbb{C}$. Combined with the fact that $H^{\prime}(\xi) \neq(n-1) \xi^{m}$ for $\xi \neq \xi_{0}$, where $\xi_{0} \in \mathbb{C}$. Hence by Lemma $2.7 H(\xi)$ is a constant, a contradiction.

If $H(\xi)=0$, and $H\left(\xi_{0}\right) \neq 0, \xi \in \mathbb{C} \backslash\left\{\xi_{0}\right\}$. Combined with the fact that $H^{\prime}(\xi) \neq(n-1) \xi^{m}$ for $\xi \in \mathbb{C} \backslash\left\{\xi_{0}\right\}$. It now follows from Lemmas 2.2 and 2.6 that this case cannot occur. Hence we show that $\mathcal{G}$ is normal at $z_{0}=0$.

Next, we show that $\mathcal{F}$ is normal at $z_{0}=0$. Since $\mathcal{G}$ is normal at $z_{0}=0$, let $g_{j} \longrightarrow g$ in a neighborhood of 0 , then there exist $\Delta_{\delta}=\{z:|z|<\delta\}$ and a subsequence of $\left\{g_{j}\right\}$ such that $\left\{g_{j}\right\}$ converges uniformly to a meromorphic function or $\infty$. Noting $g(0)=\infty$, we can find a $\varepsilon$ with $0<\varepsilon<\delta$ and $M>0$ such that $|g(z)|>M, z \in \Delta_{\varepsilon}$. So, for sufficiently large $j$, we get $\left|g_{j}(z)\right| \geq \frac{M}{2}$, hence $\left|a(z) f_{j}^{n-1}(z)\right| \leq \frac{2}{M}$. Therefore $f_{j}(z) \neq \infty$ for sufficiently large $j$ and $z \in \Delta_{\varepsilon}$.

Hence $f_{j}(z)$ is analytic in $\Delta_{\varepsilon}$. Choosing $\varepsilon$ small enough that $|a(z)| \geq \frac{|z|^{m}}{M}$, it follows that, for sufficiently large $j$, we have

$$
\left|f_{j}(z)\right|=\left|\frac{1}{a(z) g_{j}(z)}\right|^{\frac{1}{n-1}} \leq\left(\frac{2^{m+1}}{\varepsilon^{m}}\right)^{\frac{1}{n-1}}, \quad|z|=\frac{\varepsilon}{2} .
$$

By the Maximum Principle and Montel's theorem, $\mathcal{F}$ is normal at $z_{0}=0$. The complete proof of Theorem 1.1 is given.

Acknowledgements I am extremely grateful to the referee for his/her valuable suggestions and comments.

\section{References}

1. Hayman, W.K.: Research Problems of Function Theory. Athlone Press of Univ of London, London (1967)

2. Li, S.Y.: The normality criteria of a class of the functions. J. Fujian Norm. Univ. 2, 156-158 (1984)

3. Li, X.J.: Proof of Hayman's conjecture on normal families. Sci. Sin. 28, 596-603 (1985)

4. Langley, J.K.: On normal families and a result of Drasin. Proc. R. Soc. Edinb. 98A, 385-393 (1984)

5. Pang, X.C.: Bloch's principle and normal criterion. Sci. Sin. Ser. A 11, 1153-1159 (1988)

6. Chen, H.H., Fang, M.L.: On the value distribution of $f^{n} f^{\prime}$. Sci. China Ser. A 38, 789-798 (1995)

7. Bergweiler, W., Eremenko, A.: On the singularities of the inverse to a meromorphic function of finite order. Rev. Mat. Iberoam 11, 355-373 (1995)

8. Xu, Y.: Normal families of meromorphic functions. J. Math. 21(4), 381-386 (2001)

9. Zhang, Q.C.: Normal families of meromorphic functions concerning shared values. J. Math. Anal. Appl. 338, 545-551 (2008)

10. Yuan, W.J., Li, Z.R., Lin, J.M.: Some normality criteria of function families concerning shared values. Math. Methods Appl. Sci. 35(17), 2095-2100 (2012)

11. Qiu, H.L., Liu, D., Fang, M.L.: Hayman's question concerning normal families and shared values. Sci. Sin. Math. 42(6), 603-610 (2012). ((Chinese))

12. Deng, B.M., Qiu, H.L., Fang, M.L.: Hayman's question on normal families concerning zero numbers, complex variables and elliptic equations. Int. J. (2013). https://doi.org/10.1080/17476933. 2012.750307

13. Yang, J.H., Yang, Q., Pang, X.C.: A normal criterion concerning omitted holomorphic function. Acta Math. Sin. Engl. Ser. 35(12), 1972-1978 (2019)

14. Pang, X.C., Zalcman, L.: Normal families and shared values. Bull. Lond. Math. Soc. 32, 325-331 (2000)

15. Zalcman, L.: Normal families: new perspectives. Bull. Am. Math. Soc. 35, 215-230 (1998)

16. Fang, M.L.: Picard values and normality criterion. Bull. Korean Math. Soc. 38(2), 379-387 (2001)

17. Wang, Y.F., Fang, M.L.: Picard values and normal families of meromorphic functions with multiple zeros. Acta Math. Sci. 14(5), 17-26 (1998) 
18. Chang, J.M.: Normality of meromorphic functions whose derivatives have 1-points. Arch. Math. 94, 555-564 (2010)

19. Pang, X.C., Yang, D.G., Zalcman, L.: Normal families of meromorphic functions whose derivatives omit a function. Comput. Methods Funct. Theory 2(1), 257-265 (2002)

20. Chang, J.M.: Normality of meromorphic functions and uniformly discrete exceptional sets. Comput. Methods Funct. Theory 13(1), 47-63 (2013)

Publisher's Note Springer Nature remains neutral with regard to jurisdictional claims in published maps and institutional affiliations. 